\title{
Secreto Industrial y Cooperación Público-privada en I+D en el Sector Biofarmacéutico Argentino
}

\author{
Secrecy and Science-industry R\&D Cooperation in Argentine \\ Biopharmaceutical Sector
}

\author{
Vladimiro Verre', Darío Milesi², Natalia Petelski ${ }^{3}$
}

\begin{abstract}
El artículo presenta un estudio sobre el uso del secreto industrial en firmas biofarmacéuticas argentinas que innovan mediante la cooperación público-privada. Cuando la innovación se obtiene cooperando con fuentes externas y públicas de conocimiento, para la empresa aumenta el riesgo de que conocimientos críticos se filtren a potenciales competidores, debilitando la eficacia del secreto industrial. Para indagar sobre este aspecto, se han efectuado estudios de casos sobre firmas cuyos proyectos de innovación son realizados en cooperación con instituciones públicas de I+D. Los resultados obtenidos indican que las características específicas que asume la cooperación generan diferentes posibilidades a las firmas para enfrentar dichos riesgos, adaptar su estrategia de apropiación y proteger los conocimientos generados.
\end{abstract}

Keywords: innovación; cooperación en I+D; secreto industrial; apropiación; biofarmacéutico.

The article presents a study on the use of secrecy in Argentine biopharmaceutical firms that innovate through public-private cooperation. When innovation is achieved by cooperating with public and external sources of knowledge, the company is exposed to higher risks that critical knowledge leaks out to potential competitors, undermining the effectiveness of secrecy. To investigate this issue, study cases have been conducted on firms whose innovation projects are carried out by cooperating with public R\&D institutions. The results suggest that the specific features of cooperation determine different possibilities for firms to face those risks, adapt its strategy of appropriation and protect the generated knowledge.

Keywords: innovation; R\&D cooperation; secrecy; appropriation; biopharmaceutical.

\footnotetext{
Investigador docente, Instituto de Industria, Universidad Nacional de General Sarmiento (UNGS), calle J.M. Gutiérrez I I50, Los Polvorines. Provincia de Buenos Aires, Argentina, (I6I3). Phone: 0054-I I-4469-7500 (int. 7558).

E-mail: 'vverre@ungs.edu.ar, ${ }^{2}$ dmilesi@ungs.edu.ar, ${ }^{3}$ npetelsk@ungs.edu.ar
}

ISSN: 07 I8-2724. (http://www.jotmi.org) 


\section{Introducción}

La competitividad de las firmas y el mejor posicionamiento en mercados locales e internacionales residen cada vez más en su capacidad para innovar. Sin embargo para que la empresa pueda beneficiarse de las innovaciones efectuadas necesita protegerlas, apropiándose de su valor y de la renta extraordinaria asociada a ellas, previniendo que éstas sean copiadas o, si ello no fuera posible, evitando que sean desplazadas del mercado por los imitadores. La literatura sobre apropiación se centra preferentemente en los mecanismos (identificados como legales y estratégicos) que las firmas utilizan para este fin, aunque sin vincular ese uso a las características del proceso que derivó en la innovación que intentan proteger. Uno de los elementos de importancia central para la innovación, y que no es habitualmente considerado al analizar la apropiación, es la cooperación.

En este trabajo se plantea que la cooperación que desarrollan las firmas en sus actividades de innovación tiene incidencia en sus estrategias de apropiación porque, al vincularse para desarrollar procesos y productos innovadores, el conocimiento generado no queda exclusivamente en poder de la firma innovadora. En particular, cuando las firmas se vinculan y cooperan para innovar con organismos públicos como universidades, centros públicos de Investigación y Desarrollo (I+D), entre otros, parte del conocimiento generado queda en poder de actores que podrían tener como objetivo la difusión del mismo. En ese marco, puede verse particularmente afectada la eficacia del secreto industrial, un mecanismo de apropiación crítico para las firmas, cuyo uso es muy generalizado. El objetivo de este trabajo es analizar mediante un estudio de casos múltiples el efecto que tiene la cooperación con centros públicos de I+D sobre el uso del secreto industrial para proteger las innovaciones derivadas de esa cooperación. Para ello, se analiza la utilización que hacen del mecanismo del secreto industrial nueve firmas pertenecientes al sector biofarmacéutico argentino, donde una parte importante de la generación del conocimiento se apoya en la cooperación público-privada.

En la siguiente sección se presenta el marco conceptual que aborda, por una parte, la importancia de la apropiación de los resultados de la innovación para las empresas, y los mecanismos que pueden ser usados por las mismas para proteger sus innovaciones, haciendo particular hincapié en el secreto industrial $y$, por la otra, algunos aspectos relevantes de la cooperación entre actores privados productivos y actores públicos de I+D con el objetivo de innovar. La sección tres describe brevemente la metodología de investigación empleada.A partir de dicho marco conceptual y metodológico, en la sección cuatro se analizan las características que asume la vinculación público-privada en las firmas seleccionadas y se identifican diferentes "esquemas de cooperación". Pos- teriormente, se analizan las dimensiones sobre las que se apoya el uso del secreto industrial por parte de las firmas (sección cinco), para luego, sobre la base de esa evidencia, identificar algunos rasgos de la relación entre formas de cooperar y modos de implementación del secreto (sección seis). Finalmente, la sección siete sintetiza las principales conclusiones.

\section{Marco conceptual}

Para analizar el uso del secreto industrial en el sector biofarmacéutico se parte de un marco que relaciona la apropiación de los resultados de la innovación de las firmas con la generación público-privada del conocimiento. Como la estrategia de apropiación de una firma se basa en el uso de uno o más mecanismos de apropiación existentes, se hace una breve referencia a algunos de ellos, profundizando sobre las características del secreto industrial. Posteriormente se considera la cooperación público-privada como rasgo estructural de la actividad de innovación de las firmas biofarmacéuticas. Luego de identificar algunas características que puede asumir la cooperación en I+D, se analiza la relevancia de este modo de generar el conocimiento e innovar para la estrategia de apropiación y, en particular, para el uso y eficacia del secreto industrial.

\section{I. La apropiación de la innovación}

La capacidad de innovar constituye un elemento central de la competitividad de las empresas. Pero la actividad innovadora es rentable en la medida en que la empresa se beneficia de los resultados de su innovación a través de su desempeño en el mercado (Schumpeter, 1942). Para beneficiarse de los resultados de la innovación, obteniendo una renta extraordinaria respecto a sus competidores, es crucial la estrategia de apropiación que se materializa en los mecanismos y modalidades que la firma utiliza para proteger y apropiarse de los beneficios de su innovación.

Una línea creciente de literatura, iniciada por Levin et al. (1987), se ha centrado en el análisis empírico y conceptual de los mecanismos a través de los cuáles las firmas efectivamente protegen y apropian los beneficios de la innovación. De la evidencia recogida por dicho estudio seminal y numerosos trabajos posteriores en la misma línea (Harabi, 1995; Cohen et al., 2000; Arundel, 200I; Galende del Canto, 2006; González y Nieto, 2007; entre otros), se deriva que los principales mecanismos de apropiación utilizados por las firmas pueden clasificarse en dos grandes categorías: i) legales (Derechos de Propiedad Intelectual, DPI) y ii) estratégicos. Asimismo, dado que los distintos mecanismos presentan particularidades en términos de las ventajas y desventajas que aportan para proteger diferentes tipos de innovaciones, se observa que en muchos casos las firmas los utilizan en forma combinada y complementaria. 
Los mecanismos de apropiación legales se caracterizan por ser instrumentos formales que implican un registro legal de la innovación a partir del cual se otorgan derechos sobre la misma. Dentro de este grupo se encuentran las patentes, los modelos y diseños industriales, los modelos de utilidad y las licencias'. La patente consiste en un derecho exclusivo y temporal otorgado al inventor, que asegura la protección de su descubrimiento y la exclusión de terceros en la producción o uso del mismo. La patente es concebida como una solución intermedia que, por un lado, garantiza la apropiación privada de los resultados de la innovación y por lo tanto constituye un incentivo a la actividad innovadora $y$, por el otro, permite cierta difusión del conocimiento, ya que exige la descripción de la innovación en un documento accesible al público (Griliches, 1990).Varios estudios muestran que las patentes son más utilizadas para proteger las innovaciones de producto que las de proceso y que, a su vez, su uso y efectividad varían según el sector industrial (Mansfield, 1986; Levin et al., 1987; Cohen et al., 2000).

Los mecanismos de apropiación estratégicos por su parte se basan en características, conductas y acciones de las firmas orientadas a evitar o retardar la copia de sus innovaciones o a proteger el desempeño competitivo de las mismas. Entre los mecanismos estratégicos utilizados por las firmas se destacan mover primero, los activos complementarios y el secreto industrial. Mover primero implica que la empresa innovadora, en virtud de haber sido la primera en introducir una determinada innovación, logra un temprano posicionamiento en el mercado que le confiere un conjunto de ventajas productivas y comerciales difíciles de igualar por los potenciales competidores. Cuando este comportamiento se repite de forma reiterada y sistemática, puede considerarse que la firma se apropia de los resultados de su innovación a través de una conducta de 'innovación continua', que tiende a mantener constante la brecha que la separa de sus seguidores y que funciona como mecanismo de apropiación (Fernández Sánchez, 2004; Galende del Canto, 2006; Milesi et al., 20I I). Los activos complementarios, según el enfoque desarrollado por Teece (1986), son capacidades o activos que es necesario utilizar conjuntamente con el know how innovador, para una comercialización exitosa de la innovación. Los activos que Teece explícitamente identifica, y los que identifican otros autores que se apoyan en su enfoque, pueden ser divididos en dos grupos, según estén más ligados a la fase de producción o a la de comercialización (Milesi et al., 20l I). La posesión de uno o varios de estos activos puede ser esencial para que la firma logre apropi-

\footnotetext{
'De los diferentes mecanismos legales se detallan únicamente algunas características de las patentes por la importancia relativa que revisten para el sector biofarmacéutico (Coriat et al., 2003; Correa, 1990; Díaz et al., 2006; entre otros) a diferencia de los otros mecanismos legales que no son casi utilizados en esta actividad.
}

arse del resultado de su innovación en el mercado y de este modo constituir un elemento determinante en su estrategia de apropiación, especialmente en los casos en que la copia no pueda ser evitada.

Finalmente, el secreto industrial es uno de los mecanismos de apropiación estratégicos más utilizados por las empresas de todos los sectores. Su utilización implica mantener el conocimiento asociado a la innovación dentro de los límites de la empresa, para evitar que el mismo fluya hacia los competidores y sea replicable. En la literatura el secreto industrial es considerado más adecuado y funcional para proteger innovaciones de proceso en comparación con las de producto,ya que en este último caso el lanzamiento de la misma al mercado implica una difusión que dificulta el intento de mantener las características innovadoras en secreto (Harabi, 1995; Arundel, 200I; Fernández Sánchez, 2004). También el secreto industrial es usado por las firmas que focalizan sus esfuerzos en el desarrollo de innovaciones incrementales, ya sean de bienes o servicios, que quedan excluidas del régimen de patentes por no cumplir con la condición necesaria de novedad.

En distintos estudios realizados en España (González y Nieto, 2007; Galende del Canto, 2006) y Suiza (Harabi, 1995) sobre un conjunto de empresas pertenecientes a distintos sectores de la industria y/o servicios y de diferentes tamaños, el secreto industrial ha sido considerado repetidamente entre los tres primeros mecanismos en términos de uso y efectividad para proteger innovaciones $y$, en todos los casos, mostrando porcentajes significativamente superiores a los registrados por los mecanismos legales, en particular patentes $^{2}$ (Milesi et al., 20l3).

En este marco, el secreto industrial constituye un mecanismo casi natural de apropiación que consiste en no revelar información tecnológica clave acerca de las innovaciones obtenidas. Sin embargo, en términos generales, el secreto industrial presenta algunas limitaciones que derivan del hecho de que la simple acción de la empresa innovadora de no dar a conocer por sí misma esa información puede no ser suficiente para evitar de manera efectiva que la misma llegue finalmente, a través de algún mecanismo no previsto

\footnotetext{
${ }^{2} \mathrm{Si}$ bien la literatura sobre apropiación considera de manera generalizada a las patentes y al secreto industrial como mecanismos de apropiación alternativos (Levin et al, 1987, Cohen et al, 2000), hay autores que indican que esto no necesariamente es así (Hussinger, 2006; Arundel, 200 I; Brouwer y Kleinknecht, 1999). En particular, cuando la innovación recurre a fuentes externas de conocimiento (rasgo típico del sector biofarmacéutico) el secreto sigue teniendo una elevada relevancia porque, aún cuando se patente, el proceso de cooperación va generando conocimientos compartidos que pueden ser difundidos y perjudicar a la firma.
} 
o que se encuentre fuera de su control, a los competidores. En casos vinculados con la innovación de productos, una vez que estos llegan al mercado, las empresas innovadoras que solo utilizan el secreto industrial como mecanismo de protección de sus conocimientos quedan expuestas al riesgo de ingeniería reversa por parte de sus competidores (Arundel, 200I; Hussinger, 2006).

Por otra parte, para mantener la información básica asociada a una innovación en secreto una empresa debe tener en cuenta a los diferentes actores y canales a través de los cuales la misma puede filtrarse. En el ámbito interno de la firma, los trabajadores ocupan un espacio clave tanto en la generación de conocimiento como en la posibilidad de mantenerlo fuera del alcance de los competidores. Para evitar o reducir las filtraciones asociadas al personal las firmas pueden implementar tres tipos de estrategias. Una de carácter defensivo que implica algún grado de restricción a la circulación interna de la información mediante la implementación de dispositivos tales como claves de ingreso, firmas digitales, etc. que permitan limitar el acceso a determinada información a grupos reducidos de personas. La segunda se refiere a las políticas de confidencialidad, cláusulas que los trabajadores deben firmar comprometiéndose a no divulgar información crítica de la empresa. La tercera estrategia se refiere a evitar la difusión externa del conocimiento a través de la salida de trabajadores hacia empresas competidoras transfiriendo el conocimiento tácito relacionado con la innovación, el cual, por su mismo carácter constituye una ventaja central de las firmas innovadoras con respecto a posibles imitadoras. En el desarrollo de esta estrategia cobran especial relevancia las políticas de recursos humanos (Fernández Sánchez et al., 1998; Hurmelinna-Laukkanen y Puumalainen, 2007).

En la relación con los proveedores, pueden ser coherentes con la estrategia del secreto industrial prácticas tales como las de fragmentar las tercerizaciones del proceso productivo entre varios proveedores para evitar que uno solo conozca el ciclo completo que lleva a la innovación o, como señalan Fernández Sánchez et al. (1998), fabricar sus propias máquinas y de este modo evitar difundir especificaciones técnicas que develan información clave sobre la innovación y que los proveedores pueden usar o difundir entre la competencia. Los contratos de exclusividad y de confidencialidad también son relevantes en el marco de este tipo de vínculos.

Finalmente, la existencia de alianzas, acuerdos de cooperación o incluso acciones conjuntas menos formales de las empresas innovadoras con otras empresas o con instituciones, también plantean un desafío a estrategias de apropiación basadas en el secreto industrial. En este tipo de vínculos generalmente se comparte información y conocimientos relativos a la innovación por lo que surge la necesidad de evitar rupturas en las relaciones y de mantener una estabilidad de largo plazo a partir del establecimiento de condiciones contractuales (o pautas de interacción) equitativas que fomenten la confianza y consoliden la interdependencia.

\subsection{Estrategia de apropiación cuando la generación de conocimiento es público-privada}

En su abordaje sobre el uso de mecanismos de apropiación y sobre su efectividad, la literatura en general no considera la forma en que la innovación se ha obtenido. En parte, este tratamiento obedece a que se considera que las firmas no realizarían esfuerzos para innovar, o lo harían en un grado inferior al deseable, si no tuvieran alguna certeza sobre la posibilidad de apropiar sus beneficios (Arrow, 1962). Sin embargo, dado que la actividad innovadora es intrínsecamente incierta, la estrategia de apropiación se va definiendo a medida que avanza el proceso de innovación (Milesi et al., 20l3). En los casos en los que la generación de conocimientos involucra a agentes externos a la firma, en particular cuando se trata de agentes de carácter público, las posibilidades de apropiación de las innovaciones obtenidas de esa actividad cooperativa y los beneficios que las mismas generan en el mercado pueden encontrarse especialmente desafiadas. Parte del conocimiento generado permanece en el ámbito público que puede no tener incentivos para mantenerlo en secreto sino que, al contrario, puede tener interés en que el mismo se difunda, generando de esta forma el riesgo de aparición de competidores potenciales para la firma innovadora.

En actividades intensivas en conocimiento y ligadas al nuevo paradigma tecno-productivo, como es el caso del sector biofarmacéutico, las empresas recurren con frecuencia a fuentes externas de conocimiento para innovar. Por lo tanto, si se quiere analizar la estrategia de apropiación de las firmas que operan en esta industria, es necesario considerar las formas en que se genera y desarrolla esa cooperación. Para ello, se pueden considerar cuatro elementos identificados por Bercovitz y Feldman (2007) que caracterizan a las actividades de cooperación, cuya articulación es relevante para comprender la forma en que se desarrolla el proceso de innovación y el modo en que se relacionan los actores privados y públicos. En primer lugar, el origen del vínculo entre la empresa y el centro público de I+D, que puede remitir a relaciones personales o tener mayor grado de formalidad. En segundo lugar, el tipo de colaboración, que puede estar más cercano a la explotación, es decir un refinamiento y extensión de las competencias ya existentes donde las firmas acceden a un conocimiento complementario al propio, o a la exploración, orientada a la búsqueda, el descubrimiento y el desarrollo de conocimientos nuevos para la empresa, donde los resultados son más inciertos (March, 1991). En tercer lugar, debe considerarse el objeto de la colaboración, que 
puede abarcar desde proyectos complejos y de largo plazo basados en el co-desarrollo hasta proyectos más simples y de corto plazo, donde el centro de I+D puede abastecer a la empresa de un insumo específico, efectuar un servicio puntual o realizar una fase del proceso de I+D. Por último, es relevante la frecuencia de la colaboración que, dependiendo del objeto de la misma, puede ser alta u ocasional, según la cantidad de interacciones que se verifican entre las partes (Bercovitz y Feldman, 2007).

La cooperación público-privada puede presentar características variadas según el proyecto del que se trate y es posible identificar diferentes 'esquemas de cooperación', según se combinen los cuatro elementos mencionados. El esquema de cooperación puede responder a diversas situaciones, según la firma privilegie los desarrollos internos, se dirija a centros públicos de I+D solamente para servicios esenciales o apunte a proyectos complejos de co-desarrollo.

\section{Metodología}

Las firmas dedicadas a la biofarmacéutica en Argentina son 25 (Gutman y Lavarello, 2010). La presente investigación se basa en el estudio de nueve casos de empresas de dicho grupo $(36 \%)^{3}$, localizadas en la Ciudad Autónoma de Buenos Aires y sus alrededores, cuyas innovaciones biotecnológicas se realizan en cooperación con organismos públicos nacionales de I+D. La selección de los casos se realizó en base a información obtenida a través de tres fuentes: estudios sectoriales; informantes clave expertos en el sector y conocedores de la relevancia de la actividad innovadora de las firmas; e información institucional de las mismas empresas. Esta selección permitió organizar una base de trece firmas a contactar, todas ellas localizadas en el Área Metropolitana de Buenos Aires. De ese grupo, nueve tuvieron disposición para ser entrevistadas y formar parte del presente estudio.

En este marco, se llevó a cabo una investigación exploratoria y descriptiva de naturaleza cualitativa, mediante la realización de un estudio de casos múltiples (Eisenhardt 1989; Yin, 198la; b). La elección de dicha estrategia de investigación se relaciona con el tipo de pregunta de investigación planteado, que apunta a entender cómo las características de la cooperación influyen en el uso del secreto industrial. Una de las principales ventajas del abordaje cualitativo es la de permitir comprender los complejos fenómenos sociales, donde las condiciones de contexto están fuertemente relacionadas con el fenómeno estudiado (Yin, 2003). En este tipo de estudios, el objetivo de una muestra teórica

${ }^{3}$ Las firmas analizadas son: grupo Amega Biotech, Laboratorios Beta SA, Biocientífica SA, Biosidus SA, Laboratorio Pablo Cassará SRL, Laboratorio Craveri SAIC, Delta Biotech SA, Laboratorio Dosa SA y Laboratorio Elea SACIFyA. es elegir casos que pueden replicar o extender la teoría emergente (generalización analítica), sin por el contrario pretender alcanzar generalizaciones estadísticas (Eisenhardt, 1989; Yin, 2003). Por ello, en la selección de los casos se trató de incluir empresas heterogéneas en cuanto a tamaño, antigüedad y líneas de productos, para poder individualizar proyectos de cooperación biotecnológicos que respondieran a diferentes situaciones empresariales, de forma tal que fuera posible identificar diferentes patrones de cooperación y de apropiación, hasta alcanzar la 'saturación' de la teoría (Eisenhardt, 1989).

Se partió del marco teórico que orienta la investigación, el cual permitió una preidentificación de los aspectos fundamentales a indagar. El estudio se realizó a través de entrevistas en profundidad presenciales siguiendo una guía de pautas y preguntas abiertas con el Presidente de la Firma o, en su defecto, con el Director de I+D. Las entrevistas se centraron en los aspectos relativos a la cooperación con instituciones públicas para innovar, la utilización de mecanismos de apropiación de los resultados de las innovaciones, con especial atención al uso del secreto industrial y el impacto de la generación conjunta (público-privada) de conocimiento sobre la estrategia de apropiación.

Se ha optado por tomar el proyecto biotecnológico, y no la firma, como unidad de análisis, ya que una misma empresa puede llevar adelante varios proyectos que siguen lógicas y modalidades de cooperación diferentes entre sí. Las pautas seguidas tanto en la selección de las empresas a estudiar como en la elaboración del instrumento de recolección de la información, han permitido aplicar la comparación entre casos analizados, logrando la constitución de un nexo sólido entre los antecedentes teóricos iniciales y la evidencia empírica relevada, lo cual se refleja en el orden lógico de la presentación de los resultados.

\section{Generación conjunta de conocimiento en el sec- tor biofarmacéutico argentino}

Cada una de las nueve firmas estudiadas ha efectuado por lo menos un proyecto biotecnológico con instituciones públicas de I+D. Al considerar cada proyecto se han analizado las cuatro características arriba mencionadas que son críticas para describir la cooperación público-privada, es decir: origen del vínculo, tipo de cooperación, frecuencia y objeto de la misma. La identificación de diferentes situaciones ha permitido agrupar a los proyectos biotecnológicos considerados en tres formas distintas de cooperar, a las que se denomina aquí 'esquema de cooperación'. En el Cuadro I se propone una síntesis de las principales características que asume la cooperación dentro de cada esquema, indicando los proyectos incluidos en los mismos. 
Si bien dichos esquemas son lo suficientemente amplios para admitir en su interior ulteriores diferenciaciones y particularidades, reúnen requisitos mínimos para que se pueda apreciar la existencia de tres modos de cooperar que difieren claramente entre sí.A continuación, para cada esquema de cooperación, se presentan sus características principales, haciendo referencia a los rasgos relevantes de los proyectos considerados. (Cuadro I)

\section{Contratación de servicios puntuales.}

Es el caso de las empresas más pequeñas como Biocientífica, Laboratorio Dosa y Delta Biotech. Estas empresas presentan iniciativas de cooperación con centros públicos de I+D, generalmente universidades, cuyo origen se debe principalmente a contactos personales desarrollados en el ámbito académico y que se llevan a cabo por vía informal. La cooperación suele estar orientada principalmente a la explotación de capacidades existentes o a una exploración limitada, como en el caso de Laboratorio Dosa, orientada a generar capacidades internas ausentes. El objeto de la cooperación suele consistir en la realización de actividades específicas y servicios puntuales de corto plazo. La frecuencia de la cooperación suele ser baja y, en el marco de una vinculación acotada, los resultados son entregados a menudo 'llave en mano' a la firma, si bien por lo general ésta se esfuerza por aprender y replicar el conocimiento desarrollado externamente.

La firma Biocientífica fabrica reactivos de diagnóstico utilizando técnicas biotecnológicas y coopera con instituciones públicas para efectuar servicios puntuales, por ejemplo el control de calidad sobre insumos químicos, la realización de ensayos sobre animales en bioterios y el desarrollo de productos puntuales. Laboratorio Dosa fabrica medicamentos tradicionales y actualmente está incursionando en la biotecnología con la producción de filgrastim (proteína recombinante de uso oncológico). Para ello ha encargado a una universidad pública el desarrollo de un proceso productivo innovador de dicha proteína. Una vez finalizado el proyecto los conocimientos fueron transferidos a la firma que posteriormente emprendió la fase de escalado industrial. Delta Biotech produce principios activos de alta potencia para la industria farmacéutica utilizando técnicas biotecnológicas en los procesos productivos. La firma ha llegado a un proceso innovador cuyas etapas químicas, en parte fueron desarrolladas internamente $y$ en parte externamente por distintas instituciones públicas, de modo tal que solamente la firma posee el conocimiento de la secuencia completa de ese proceso específico. Delta Biotech ha extendido su innovación

\begin{tabular}{|c|c|c|c|}
\hline & \multicolumn{3}{|c|}{ ESQUEMA DE COOPERACIÓN } \\
\hline & $\begin{array}{l}\text { CONTRATACIÓN } \\
\text { DE SERVICIOS PUN- } \\
\text { TUALES }\end{array}$ & INTERNALIZACIÓN & COORDINACIÓN \\
\hline Origen del vínculo & Personal & Personal/formal & Formal \\
\hline Tipo de cooperación & $\begin{array}{l}\text { Tercerización de fases } \\
\text { (explotación) }\end{array}$ & $\begin{array}{l}\text { Creación de capacidades nuevas } \\
\text { para la firma (exploración) }\end{array}$ & $\begin{array}{l}\text { Creación de capacidades nuevas } \\
\text { para la firma (exploración) }\end{array}$ \\
\hline $\begin{array}{l}\text { Objeto de la } \\
\text { cooperación }\end{array}$ & $\begin{array}{l}\text { Realización de fases } \\
\text { específicas de corto pla- } \\
\text { zo, entregadas 'llave en } \\
\text { mano' }\end{array}$ & $\begin{array}{l}\text { Proyectos complejos de largo pla- } \\
\text { zo, con incorporación de RRHH } \\
\text { públicos }\end{array}$ & $\begin{array}{l}\text { Proyectos complejos de largo } \\
\text { plazo, basados en el co-desarrollo }\end{array}$ \\
\hline $\begin{array}{l}\text { Frecuencia de la } \\
\text { cooperación }\end{array}$ & $\begin{array}{l}\text { Baja (Delta Biotech: } \\
\text { media) }\end{array}$ & $\begin{array}{l}\text { Media (disminuye a raíz de la } \\
\text { endogeneización de la fuente de } \\
\text { conocimiento externa) }\end{array}$ & Alta \\
\hline Casos & $\begin{array}{l}\text { Biocientifica; } \\
\text { Delta Biotech; } \\
\text { Dosa }\end{array}$ & $\begin{array}{l}\text { Beta; } \\
\text { Biosidus: tambo farmacéutico fase } \\
\text { II; } \\
\text { Craveri: ingeniería de tejidos }\end{array}$ & $\begin{array}{l}\text { Amega Biotech; } \\
\text { Biosidus: tambo farmacéutico } \\
\text { fase I, terapia génica; } \\
\text { Cassará; } \\
\text { Craveri: vacuna oncológica; } \\
\text { Elea-Chemo }\end{array}$ \\
\hline
\end{tabular}

Cuadro I. Características de los esquemas de cooperación identificados. Fuente: elaboración propia

ISSN: 07 I8-2724. (http://www.jotmi.org)

Journal of Technology Management \& Innovation (C) Universidad Alberto Hurtado, Facultad de Economía y Negocios. 
de proceso a otros productos y en la fase de desarrollo ha aplicado el mismo esquema, es decir, apoyarse en una red de proveedores de bloques de etapas parciales.

\section{Internalización.}

Es el caso de Laboratorios Beta, Laboratorio Craveri (ingeniería de tejidos) y Biosidus (tambo farmacéutico). En este esquema las firmas están involucradas en proyectos de más largo alcance, en los que tratan de realizar el core de sus actividades de innovación internamente, pero recibiendo aportes fundamentales del sector público. Si bien el origen del vínculo puede remitir a contactos personales, la cooperación suele efectuarse con mayor nivel de formalidad que en el caso del esquema anterior. El tipo de cooperación suele ser exploratorio y orientado a crear conocimientos nuevos que suelen ser empleados posteriormente para ampliar la gama de productos. Dada la naturaleza experimental de dichos proyectos de cooperación, el objeto de la misma es complejo y de largo plazo. Sin embargo, en este esquema juega un rol fundamental la incorporación a la firma de recursos humanos provenientes de aquellas instituciones públicas con las que se coopera, por lo tanto la frecuencia de la cooperación es variable y puede verificarse una moderación de la misma, en paralelo con una intensificación de las actividades desarrolladas in house (a partir de la internalización de los recursos humanos provenientes de la institución pública).

Laboratorios Beta es una empresa farmacéutica de larga trayectoria en Argentina y con amplia experiencia en lo que se refiere a insulina. La firma ha incursionado en la biotecnología apuntando a la producción de insulina recombinante humana. Para ello, luego de intentar generar capacidades in house, la empresa se dirigió a un instituto público de I+D, el Instituto de Biología y Medicina Experimental (IByMECONICET) y decidió incorporar, en relación de dependencia, a un grupo de investigadores biotecnólogos de dicha institución, quienes posteriormente realizaron importantes innovaciones de proceso que posibilitaron el avance del proyecto hasta la construcción de una planta piloto. Laboratorio Craveri también es una empresa de larga trayectoria en el país, especializada en tejidos humanos. La firma mantenía relaciones de cooperación con el Instituto Leloir en temas de piel y, cuando decidió emprender un proyecto de ingeniería genética en tejidos, lo hizo incorporando en relación de dependencia a un grupo de investigadores de aquel instituto, que con el tiempo lograron realizar innovaciones que permitieron ampliar la gama de tejidos producidos. Finalmente, Biosidus es una de las empresas biotecnológicas más antiguas y renombradas de América Latina y buena parte de su prestigio está ligado al proyecto 'tambo farmacéutico' (que consiste en utilizar animales como biorreactores, es decir que produzcan en su leche proteínas recombinantes, como la insulina o la hormona de crecimiento humano). Dicho proyecto comenzó con un esquema co-participativo que involucraba a distintas instituciones públicas de $I+D$, pero posteriormente la empresa optó por internalizar el proyecto, incorporando en relación de dependencia a importantes recursos humanos de aquellas instituciones públicas que habían participado de la primera fase y apoyándose en centros públicos de I+D para servicios críticos.

\section{Coordinación.}

Es el caso del grupo Amega Biotech, Laboratorio Pablo Cassará, Biosidus (terapia génica), Laboratorio Craveri (vacuna oncológica) y Laboratorio Elea. Este esquema suele darse en el marco alianzas estratégicas que se forman entre las firmas y las instituciones públicas de I+D. El origen del vínculo suele estar caracterizado por un mayor nivel de formalidad que en los otros dos esquemas identificados y es frecuente que sea la institución pública la que propone los proyectos a la firma. El tipo de cooperación está fuertemente orientado a la exploración, los proyectos son altamente inciertos y experimentales y la generación de conocimiento presenta un elevado grado de novedad. El objeto de la cooperación es complejo y de largo plazo y suele verificarse una división del trabajo cercana al co-desarrollo, con mayor nivel de coparticipación respecto a los esquemas anteriores y con resultados compartidos. Dada la presencia de proyectos de largo plazo la innovación conjunta se basa en una interacción continua, por ende la frecuencia de la cooperación entre los actores suele ser alta.

Amega Biotech es un grupo empresario que ha establecido una alianza estratégica con el Laboratorio de Cultivos Celulares de la Universidad Nacional del Litoral (LCC-UNL). Una de las firmas del grupo, Zelltek, tiene su equipo de I+D localizado en el mismo espacio físico del LCC-UNL, lo cual ha generado un ambiente amplio, mixto y prolífico en generación de conocimientos, donde hay cierta flexibilidad en los límites entre empresa e institución, que permite constantes flujos de recursos humanos y conocimientos. En el marco de esa alianza estratégica se llevan a cabo numerosos proyectos donde se sigue un esquema de co-desarrollo y se comparten los resultados y las patentes generadas. El Laboratorio Pablo Cassará tiene una estrecha relación con la Fundación Cassará, un centro privado de I+D donde funciona el Centro Milstein, que es público-privado. La firma ha emprendido numerosos proyectos con la Fundación, por ejemplo, la producción de proteínas recombinantes y de vacunas. También en este caso hay flexibilidad entre los límites de la empresa y la institución, y la firma se encuentra en un lugar privilegiado para detectar nuevas ideas y seleccionar proyectos a desarrollar en distintas áreas del conocimiento. Los proyectos siguen la lógica del co-desarrollo y los start ups que eventualmente surgen de la Fundación suelen tener 
a la firma por socia mayoritaria. Biosidus emprendió, junto a la Fundación Favaloro, un proyecto sobre terapia génica (que consiste en la inserción de copias funcionales de genes defectuosos o ausentes en el genoma de un individuo, en este caso para favorecer la revascularización). Dicho proyecto se basó en una división del trabajo horizontal, donde Biosidus se dedicó a la construcción genética y a los aspectos regulatorios y la Fundación Favaloro a los ensayos sobre animales, la investigación clínica y la suministración a pacientes. Los resultados fueron compartidos y las patentes generadas fueron de titularidad conjunta. Laboratorio Craveri, que buscando consolidar sus capacidades endógenas en ingeniería de tejidos había incorporado un grupo de investigadores del Instituto Leloir, emprendió con la misma institución otro proyecto, una vacuna oncológica basada en la terapia génica. Este proyecto se basó en una efectiva división del trabajo (el Laboratorio se ocupó del cultivo de células primarias y el Instituto del manejo de vectores virales), en el marco de un proyecto donde primó la interacción constante y los resultados fueron compartidos. Finalmente el Laboratorio Elea, firma de importante trayectoria en Argentina, actualmente está llevando a cabo, entre otros, un proyecto sobre vacunas oncológicas en avanzado estado de desarrollo y otro, aún incipiente, para producir anticuerpos monoclonales. Lo que caracteriza ambos proyectos es que se llevan adelante con una serie de instituciones públicas y privadas que actúan como un 'consorcio' y donde se trabaja en red. Dicha red se basa en una división del trabajo articulada y las instituciones públicas que participan en la misma realizan tareas que van desde las investigaciones básicas hasta la parte clínica. Entre ellas, la institución pública que funciona como centro de I+D de la red es el Instituto de Oncología Molecular de la Universidad Nacional de Quilmes.

\section{El mecanismo del secreto industrial en la gene- ración conjunta de conocimiento}

El mecanismo del secreto industrial además de ser muy difundido entre las firmas, puede ser implementado a través de diversas acciones y puede estar dirigido tanto hacia adentro de la empresa como hacia fuera de la misma. En los casos empresariales estudiados la atención se ha centrado sobre el frente externo del secreto industrial, ya que las instituciones públicas de I+D con las que las firmas cooperan son fuentes externas de conocimiento que funcionan en algunos casos como proveedores y en otros como partners del proyecto de innovación, y el conocimiento co-generado se encuentra tanto dentro de la firma como en dichas instituciones.

El análisis de la utilización del mecanismo del secreto industrial por parte de las nueve firmas estudiadas permite identificar diferentes situaciones, donde se observa que el uso del mismo en relación a las instituciones públicas de I+D tiene dos dimensiones principales: una pasiva y otra activa.
La primera se refiere a factores contextuales que hacen poco probable que la contraparte pública ponga en peligro la efectividad del secreto industrial como mecanismo de apropiación implementado por las firmas. Dentro de la dimensión pasiva del secreto industrial hay tres factores, indicados por casi todas las firmas estudiadas, que pueden desincentivar los comportamientos oportunistas y jugar en favor de este mecanismo. En primer lugar, la naturaleza de las instituciones con las que se coopera que, al ser públicas y académicas, hace poco probable que las mismas decidan orientarse a una experiencia empresarial y productiva. En segundo lugar, quien decida eventualmente crear una nueva firma competidora debe enfrentar importantes barreras a la entrada, como la necesidad de efectuar costosas inversiones y de contar con activos complementarios críticos, entre ellos el know how empresarial, que son considerados por las firmas entrevistadas casi tan importantes como las innovaciones realizadas. En tercer lugar, hay en general un importante nivel de confianza en la contraparte que, en algunos casos, responde a relaciones personales construidas en el tiempo, pero en otros, refleja la existencia de una alianza estratégica formal entre los actores públicos y privados, que se ha consolidado a través de múltiples proyectos complejos y de largo plazo, siendo Laboratorio Elea, Laboratorio Pablo Cassará y Amega Biotech los ejemplos más notables.

Los factores contextuales que caracterizan a la dimensión pasiva del secreto industrial sirven de apoyo a la segunda dimensión, la activa. Dentro de esta dimensión pueden identificarse distintas medidas o acciones estratégicas que las firmas biofarmacéuticas adoptan para implementar el secreto industrial. En primer lugar los contratos y las cláusulas de confidencialidad, que son un instrumento muy difundido y generalizado entre las firmas. Los contratos suelen describir detalladamente de quién es la propiedad intelectual del conocimiento previo y el que se genera durante la cooperación. Sin embargo en general, las empresas usan este instrumento con escasa seguridad acerca de su eficacia real (solamente Amega Biotech se ha mostrado optimista acerca de la eventual posibilidad de que dichos contratos sean judicializables). Otro modo de fortalecer el secreto industrial consiste en la incorporación de recursos humanos que provienen de las instituciones públicas con las que se coopera. Desde esta óptica puede considerarse la incorporación de investigadores del IByME tanto por parte de Laboratorios Beta en el proyecto de insulina recombinante como por Biosidus en el proyecto del tambo farmacéutico, así como también la de los investigadores del Instituto Leloir de Laboratorio Craveri.A menudo la incorporación de recursos humanos responde a dos objetivos paralelos, es decir, crear capacidades en la firma e internalizar la fuente externa de conocimiento y, a la vez, evitar que el conocimiento clave generado quede fuera de la firma. De esta forma lo que se logra es endogeneizar el riesgo, trasladando el mismo del frente externo del secreto industrial al frente interno. 
Una tercera forma de mantener el secreto industrial frente a las instituciones públicas de I+D consiste en la fragmentación del conocimiento. Es el caso de Delta Biotech, cuyo proceso productivo innovador consiste de numerosas etapas de síntesis química, algunas de las cuales son desarrolladas internamente, mientras que en otras se contrata su desarrollo a varios centros públicos de I+D, de modo que ninguna institución conozca la secuencia completa del proceso innovador.

Una cuarta forma de fortalecer la efectividad del mecanismo del secreto industrial es estrechando alianzas estratégicas con la institución pública de I+D con la que se coopera. Cuando la cooperación evoluciona desde meras colaboraciones esporádicas y ad hoc a proyectos complejos de co-desarrollo puede entreverse la existencia de una alianza estratégica entre la firma y la institución pública, que puede abarcar un proyecto biotecnológico concreto (Biosidus y Laboratorio Elea) o múltiples proyectos (Laboratorio Pablo Cassará y Amega Biotech). En estos proyectos de largo plazo se suelen generar patentes de titularidad compartida entre la parte privada y la pública, lo cual contribuye a generar un clima de confianza que tiene efectos sobre el conocimiento protegido a través del secreto industrial, ya que en el marco de dicha alianza crecen los incentivos a la confianza mutua y a evitar comportamientos oportunistas. Este aspecto se diferencia de la confianza de la dimensión pasiva, en la que se apoyan todas las firmas estudiadas y que constituye el elemento que permite la existencia de la cooperación, ya que representa su fortalecimiento formal a través de una alianza de largo plazo.

Finalmente, un modo adicional de fortalecer el mecanismo del secreto industrial consiste en la vigilancia sobre la generación de conocimiento de centros públicos de I+D, representado por la experiencia de Amega Biotech y Laboratorio Pablo Cassará. Esta quinta forma de favorecer el secreto industrial puede surgir en el marco de alianzas estratégicas que abarcan múltiples proyectos complejos emprendidos por la firma y la institución pública, que comparten recursos humanos y espacios físicos. En el caso de Amega Biotech, hay una importante circulación de recursos hu- manos desde la empresa hacia el LCC-UNL y viceversa, en el marco de nuevos proyectos innovadores. En el caso de Laboratorio Pablo Cassará, la firma apoya abiertamente la incubación de start ups que emerjan de la Fundación Cassará, constituyéndose en socia mayoritaria de los mismos. En dichos casos, al compartir el mismo espacio físico con investigadores del sector público y habiendo cierta flexibilidad y superposición entre recursos humanos privados y públicos, las firmas logran detectar con rapidez la presencia de vocaciones emprendedoras y proyectos atractivos, que son apoyados y encauzados. Cuando el nuevo conocimiento generado está relacionado o es complementario al que ya posee la firma o en los casos en los cuales la situación de su propiedad es ambigua, la firma puede, en cierta forma, anticiparse a la salida de ese conocimiento. De tal modo se evita que el nuevo conocimiento, generado en un ambiente público-privado, fluya hacia un competidor ya existente o hacia un nuevo competidor (como un start up), lo cual podría poner en riesgo conocimientos críticos que la firma protege a través del secreto industrial.

El Cuadro 2 sintetiza los elementos que constituyen las dos dimensiones consideradas del secreto industrial de acuerdo a la evidencia analizada.

\section{El secreto industrial en los esquemas de cooper- ación}

A partir de las tres formas de cooperar identificadas y de la variabilidad relevada en el uso del secreto industrial entre las firmas analizadas, es posible evidenciar para cada esquema de cooperación, los modos de implementación predominantes del secreto industrial como estrategia de apropiación de los conocimientos generados conjuntamente, tal como se estiliza en el Cuadro 3.

Se observa que en el esquema de contratación de servicios puntuales las firmas se apoyan principalmente en la dimensión pasiva del secreto y en los contratos de confidencialidad. En el esquema de internalización las firmas absorben recursos humanos públicos con el doble objetivo de crear/ consolidar capacidades y proteger el conocimiento en el-

\begin{tabular}{|l|l|}
\hline Dimensión PASIVA & Dimensión ACTIVA \\
\hline Naturaleza de las instituciones & Contratos de confidencialidad \\
\hline Barreras a la entrada & Incorporación de RRHH públicos a la firma \\
\hline Confianza en la contraparte & Fragmentación del conocimiento \\
\hline & Constitución de alianzas estratégicas \\
\hline & Vigilancia sobre la creación de start ups \\
\hline
\end{tabular}

Cuadro 2. Las dimensiones del secreto industrial. Fuente: elaboración propia

ISSN: 07I 8-2724. (http://www.jotmi.org)

Journal of Technology Management \& Innovation (c) Universidad Alberto Hurtado, Facultad de Economía y Negocios. 
los incorporado. De este modo trasladan el riesgo de copia desde el frente externo al frente interno de la firma, confiando en la eficacia de la dimensión pasiva del secreto y de los contratos de confidencialidad. Las firmas que adoptan el esquema de coordinación, además de apoyarse en la dimensión pasiva del secreto y utilizar instrumentos de uso generalizado, como los contratos de confidencialidad, cuentan con la posibilidad de beneficiarse de otras medidas, como la constitución de alianzas estratégicas formales $y$, en algunos casos, la vigilancia y el control de la generación de start ups. En un sector intensivo en la utilización de patentes, vale la pena remarcar que todas las firmas estudiadas manifestaron la importancia de preservar la efectividad del secreto industrial como mecanismo de apropiación, en un contexto donde la innovación es generada a través de la cooperación con instituciones públicas de I+D. Este hecho se pone en evidencia al considerar que, para alcanzar dicho objetivo, en todos los esquemas de cooperación las firmas se apoyan tanto en elementos contextuales (pasivos) como en medidas activas, tales como los contratos de confidencialidad. Adicionalmente, los casos estudiados muestran que las firmas utilizan distintos métodos para fortalecer su estrategia de uso del secreto industrial, por ejemplo, en la contratación de servicios puntuales a través de la fragmentación del conocimiento entre los proveedores de esos servicios y en el esquema de internalización, a través de la endogeneización del conocimiento externo. Sin embargo el esquema de coordinación, que es el más abierto y co-participativo, parece también ser el más efectivo en moderar los riesgos de la cooperación para el uso del secreto industrial, ya que fo- menta alianzas estratégicas que dan un contexto formal a la mera 'confianza en la contraparte' de la dimensión pasiva y permite la adopción de medidas creativas, como la vigilancia sobre la generación de start ups que, además de fortalecer el secreto, posibilita el aprovechamiento de eventuales nuevos proyectos.

\section{Conclusiones}

En el presente trabajo se han analizado nueve casos de empresas pertenecientes al sector biofarmacéutico argentino cuyos proyectos biotecnológicos son realizados en cooperación con instituciones públicas de I+D. En estos casos el conocimiento innovador, en mayor o menor medida, es generado conjuntamente entre actores públicos y privados. Esta situación conlleva para la firma riesgos adicionales a los ya existentes para la estrategia de apropiación de los resultados de sus innovaciones, siendo el secreto industrial y su capacidad de ser efectivo en evitar la copia, el aspecto más expuesto a dichos riesgos. Si bien existe la percepción de que la cooperación público-privada puede afectar el uso por parte de las firmas del secreto industrial, la presente investigación se ha centrado en un aspecto poco estudiado en la literatura existente, es decir, la incidencia de la forma concreta de cooperar en I+D sobre el modo en que las firmas implementan el secreto industrial.

En un sector intensivo en la generación de patentes, como el biofarmacéutico, el uso del secreto industrial no solamente es recurrente, sino que parece ocupar un rol relevante en la

\begin{tabular}{|c|c|c|c|c|}
\hline \multirow{2}{*}{\multicolumn{2}{|c|}{$\begin{array}{c}\text { CONTRATACIÓN } \\
\text { DE SERVICIOS PUNTUALES }\end{array}$}} & \multicolumn{3}{|c|}{ ESQUEMA DE COOPERACIÓN } \\
\hline & & \multirow{2}{*}{$\begin{array}{c}\text { INTERNAL- } \\
\text { IZACIÓN } \\
\mathrm{X} \\
\end{array}$} & \multirow{2}{*}{$\frac{\text { COORDINACIÓN }}{\mathrm{X}}$} & \multirow[b]{2}{*}{$\mathrm{X}$} \\
\hline Dimensión & $\begin{array}{l}\text { Naturaleza de las insti- } \\
\text { tuciones }\end{array}$ & & & \\
\hline & Barreras a la entrada & $\mathrm{X}$ & $\mathrm{X}$ & $\mathrm{X}$ \\
\hline industrial & $\begin{array}{l}\text { Confianza en la contra- } \\
\text { parte }\end{array}$ & $\mathrm{X}$ & $\mathrm{X}$ & $\begin{array}{l}\mathrm{X} \text { (alianza es- } \\
\text { tratégica) }\end{array}$ \\
\hline \multirow{5}{*}{$\begin{array}{c}\begin{array}{c}\text { Dimensión } \\
\text { ACTIVA }\end{array} \\
\text { del secreto } \\
\text { industrial }\end{array}$} & $\begin{array}{l}\text { Contratos de confidencial- } \\
\text { idad }\end{array}$ & $\mathrm{X}$ & $\mathrm{X}$ & $\mathrm{X}$ \\
\hline & $\begin{array}{l}\text { Incorporación de RRHH } \\
\text { públicos a la firma }\end{array}$ & & $\mathrm{X}$ & \\
\hline & $\begin{array}{l}\text { Fragmentación del cono- } \\
\text { cimiento }\end{array}$ & $\mathrm{X}$ & & \\
\hline & $\begin{array}{l}\text { Constitución de alianzas } \\
\text { estratégicas }\end{array}$ & & & $\mathrm{X}$ \\
\hline & $\begin{array}{l}\text { Vigilancia sobre la } \\
\text { creación de start ups }\end{array}$ & & & $\mathrm{X}$ \\
\hline
\end{tabular}

Cuadro 3. El secreto industrial en los tres esquemas de cooperación. Fuente: elaboración propia

ISSN: 07 I8-2724. (http://www.jotmi.org)

Journal of Technology Management \& Innovation (c) Universidad Alberto Hurtado, Facultad de Economía y Negocios. 
estrategia de apropiación de las firmas estudiadas. Partiendo de un marco conceptual que conjuga apropiación y cooperación público-privada, es posible identificar tres esquemas de cooperación, en cada uno de los cuales los efectos potencialmente negativos que la cooperación público-privada puede ejercer sobre el secreto industrial, son tamizados de forma diferente.

Como surge de la evidencia analizada, los esquemas de contratación de servicios puntuales e internalización fortalecen el secreto industrial con algún grado de reducción de los alcances de la cooperación, diversificando estratégicamente las tercerizaciones o endogeneizando conocimientos externos críticos respectivamente, ya que la firma apunta fundamentalmente al fortalecimiento de sus capacidades endógenas de innovación. El esquema de coordinación, por el contrario, se caracteriza por lograr combinar proyectos altamente innovadores y potencialmente muy rentables con una horizontalidad estructural entre los actores. En este contexto el uso del secreto industrial cobra una mayor relevancia ya que la alianza estratégica prima sobre las medidas defensivas de los otros esquemas y consolida un contexto donde es posible incluso transformar un riesgo potencial en el aprovechamiento posterior del conocimiento co-generado. En este esquema, el riesgo de que el conocimiento co-generado quede en la parte pública y pueda ser difundido, pierde transcendencia relativa, $y$ prácticamente se desvanece, como consecuencia de la consolidación de un ambiente de interacción público-privado basado en la constante circulación de personas, recursos e ideas, donde las firmas pueden encontrar nuevos estímulos para innovar.

\section{Referencias}

ARROW, K. (1962) Economic Welfare and the Allocation of Resources for Invention. En: R. Nelson (ed.), The Rate and Direction of Inventive Activity. Princeton University Press, Princeton (NJ). pp. 609-625.

ARUNDEL, A. (200I). The Relative Effectiveness of Patents and Secrecy for Appropriation. Research Policy, 30(4), 6I I624.

BERCOVITZ, J. y Feldman, M. (2007). Fishing upstream: Firm innovation strategy and university research alliances. Research Policy, 36(7), 930-948.

BROUWER, E. y Kleinknecht, A. (1999). Innovative output and a firm's propensity to patent. An exploration of CIS micro data. Research Policy, 28(6), 615-624.

COHEN, W.M.; Nelson, R.r. Y Walsh, J.p. (2000). Protecting Their Intellectual Assets: Appropriability Conditions and Why Us Manufacturing Firms Patent (or Not). National Bureau of Economic Research, Cambridge (MA).

CORIAT, B., Orsi, F.Y Weinstein, O. (2003). Science- Based Technological Regimes and Institutions: Does Biotech Reflect a New Science Based Regime? Industry and Innovation I0(3), 23I-253.

CORREA, C.M. (1990). Patentes, industria farmacéutica y biotecnología. Revista del Derecho Industrial, 12, 335-343.

DÍAZ,A.; Krimer, A.Y Medina, D. (2006) Salud Humana: de la industria farmacéutica a los biofármacos. En: Bisang, R.; Gutman, G.; Lavarello, P.; Sztulwark, S.Y Díaz, A. (Comp.). Biotecnología y desarrollo. Un modelo para armar en la Argentina. Prometeo Libros, Buenos Aires. pp. 103- 138.

EISENHARDT, K. M. (1989). Building Theories from Case Study Research. Academy of Management Review, 14(4), 532-550.

FERNÁNDEZ Sánchez, E.; Montes Peón, J.m.; Pérez Bustamante, G. Y Vázquez Ordás, C.j. (1998). Acumulación, Naturaleza e Imitación del Conocimiento Tecnológico: una Revisión de la Literatura. Investigaciones Europeas de Dirección y Economía de la Empresa, 4(I), II-34.

FERNÁNDEZ Sánchez, E. (2004). Formas de apropiación de las ganancias de una innovación. Universia Business Review, I, 70-8I.

GALENDE Del Canto, J. (2006). La apropiación de los resultados de la actividad innovadora. Manuscrito no publicado, Universidad de Salamanca.

ISSN: 07I 8-2724. (http://www.jotmi.org) 
GONZÁLEZ, N. y Nieto, M. (2007). Appropriability of innovation results: An empirical study in Spanish manufacturing firms. Technovation, 27(5), 280-295.

GRILICHES, Z. (1990). Patent Statistics as Economic Indicators: A Survey. Journal of Economic Literature, 28(4), I66I1707.

GUTMAN, G. y Lavarello, P. (20I0). Desarrollo reciente de la moderna biotecnología en el sector de salud humana. Documento del Proyecto Ceur-Conicet "Potencialidades de la biotecnología para el desarrollo industrial de Argentina", Buenos Aires.

HARABI, N. (1995). Appropriability of technical innovations: An empirical analysis. Research Policy, 24(6), 98I-992.

HURMELINNA-LAUKKANEN, P. y Puumalainen, K. (2007). Nature and Dynamics of Appropriability: Strategies for Appropriating Returns on Innovation. R\&D Management, 37(2), $95-112$.

HUSSINGER, K. (2006). Is Silence Golden? Patents versus Secrecy at the Firm Level. Economics of Innovation and New Technology I5(8), 735-752.

LEVIN, R.C.; Klevorick,A.k.; Nelson, R.r.;Winter, S.g.; Gilbert, R.Y Griliches, Z. (1987). Appropriating the Returns from Industrial Research and Development. Brookings papers on economic activity, I8(3), 783-83I.

MANSFIELD, E. (1986). Patents and Innovation: an empirical study. Management Science, 32(2), I73-I8I.

MARCH, J. (|99|). Exploration and exploitation in organizational learning. Organization Science, 2(I), 7I-87.

MILESI, D.; Verre, V.; Petelski, N.Y Aggio, C. (20I I). Apropiación privada de las rentas de la innovación: elementos para la discusión conceptual y el abordaje metodológico. Manuscrito no publicado, Universidad Nacional de General Sarmiento.

MILESI, D., Petelski, N., Verre, V., (20I3). Innovation and appropriation mechanisms: Evidence from Argentine microdata.Technovation, 33(2-3), 78-87.

SCHUMPETER, J.A. (1942). Capitalism Socialism and Democracy. Harper and Row, New York.

TEECE, D.J. (1986). Profiting from Technological Innovation: Implications for Integration, Collaboration, Licensing and Public Policy. Research policy, I5(6), 285-305.
YIN, R. (198Ia), The case study as a serious research strategy. Knowledge: Creation, Diffusion, Utilization, 3, 97-II 4.

YIN, R. (198Ib). The case study crisis: Some answers. Administrative Science Quarterly, 26, 58-65.

YIN, R. (2003). Case study research: design and methods. Third edition:Applied Social Research Methods Series, Vol. 5. SAGE Publications, Thousand Oaks, CA. 